\title{
Sovrascrizione e sovrascrittura
}

\author{
Valeria Della Valle
}

PUBBLICATO: 25 LUGLIO 2019

\section{Quesito:}

Alcuni lettori ci chiedono ragguagli intorno ai termini sovrascrizione e sovrascrittura usati in testi di argomento informatico.

\section{Sovrascrizione e sovrascrittura}

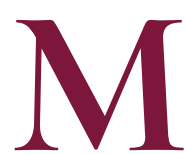

entre il verbo sovrascrivere è registrato da tempo in alcuni vocabolari della lingua italiana, con definizioni che fanno riferimento sia al non comune "scrivere sopra", sia al significato relativo all'uso informatico (Garzanti 2008 spiega: "Sovrascrivere un file, sostituirlo con un altro file che ha lo stesso nome ma contiene dati più aggiornati", e Zingarelli 2or8: "Copiare una porzione di testo o un file cancellando i dati preesistenti"), nessun repertorio lessicografico ha registrato, finora, i sostantivi sovrascrizione e sovrascrittura, nonostante siano termini utilizzati frequentemente dagli informatici.

Unici a essere registrati sono i lemmi di antica data soprascrittura (riportato dal GDLI col significato di 'introduzione, esordio, intestazione di una lettera', con la citazione di un esempio secentesco di Paolo Sarpi) e soprascrizione, risalente come prima attestazione alla fine del XIII secolo (riportato fin dalla prima edizione del Vocabolario degli Accademici della Crusca, dal GDLI e dal GRADIT con i significati di 'inscrizione', 'iscrizione su una moneta', 'epitaffio', 'soprascritta', 'contrassegno').

Se sovrascrizione e sovrascrittura nel significato informatico sono ignorati dai vocabolari della lingua italiana, anche negli archivi dei quotidiani se ne trovano scarsissime tracce. Nessuna citazione di sovrascrizione è presente negli archivi della "Repubblica", del "Corriere della sera" e della "Stampa". Diverso il caso di sovrascrittura e soprascrittura le cui citazioni, però, fanno riferimento ad altro significato, cioè quello di 'scritta tracciata sopra un'altra' (in una scheda elettorale, in un registro, in un'epigrafe, in un testo teatrale). In un unico un contesto giornalistico sovrascrittura fa riferimento al suo valore in informatica:

Un ostacolo alla ricostruzione di quanto è stato "cancellato" può derivare dalla deframmentazione, vera e propria risistemazione dei dati e loro sovrascrittura sul disco rigido (ovviamente correlata da opportune modifiche del file-system). (Ornella Rota, "la Stampa", I9 maggio 2004, Tuttoscienze, p. 2)

L'interrogazione in Google dà 3.6oo risultati per sovrascrizione, 6.650 per soprascrizione (ma sempre nel significato di antica data riportato sopra), , Io.oooo, invece, per sovrascrittura. Alla luce di questi dati sovrascrittura sembra imporsi, numericamente, sul meno comune sovrascrizione, che viene segnalato come forma non accettabile dal correttore. Anche questa censura correttoria automatica ha contribuito, probabilmente, a far preferire e specializzare sovrascrittura rispetto a sovrascrizione nel significato relativo all'uso informatico. Sempre in rete, è frequentissima l'espressione "modalità sovrascrittura", e per sovrascrittura si diche che "consiste nel salvataggio di un nuovo file su settori logici impegnati da elementi precedenti". Si tratta, dunque, di termine che non potrà più mancare (insieme al meno comune sovrascrizione) nei vocabolari del futuro. 


\section{Cita come:}

Valeria Della Valle, Sovrascrizione e sovrascrittura , "Italiano digitale", 2019, X, 2019/3 (lugliosettembre)

DOI: $10.35948 / 2532-9006 / 2020.3198$

Copyright 2019 Accademia della Crusca

Pubblicato con licenza creative commons CC BY-NC-ND 